\title{
An End Theorem for Stratified Spaces
}

\author{
Frank Connolly Bogdan Vajiac
}

February, 1996

\begin{abstract}
We show that a tame ended stratified space $X$ is the interior of a compact stratified space if and only if a K-theoretic obstruction $\gamma_{*}(X)$ vanishes. The obstruction $\gamma *(X)$ is a localization of Quinn's mapping cylinder neighborhood obstruction. The main results are Theorem 1.6 and Theorem 1.7 below. In particular, this explains when a $G$-manifold is the interior of a compact $G$-manifold with boundary. Our methods include a new transversality theorem, Corollary 1.17.
\end{abstract}

\section{Main Results, Background and Definitions.}

1.1. A beautiful theorem of geometric topology characterizes those topological manifolds which can be the interiors of compact manifolds with boundary. The breakthrough in this direction, [1], due to Browder, Levine and Livesay, states that a smooth manifold $M^{n}$ is the interior of a compact smooth manifold with simply connected boundary provided only that $n \geq 6, H_{*}(M, \mathbb{Z})$ is finitely generated, and $M$ is simply connected at infinity. This was promptly generalized by L. Siebenmann in [17], and in [7]. He showed that a tame ended topological manifold $X^{n}$ is the interior of a compact manifold with boundary if and only if an obstruction $\sigma(X)$, in $\tilde{K}_{0}(\mathbb{Z} \pi)$, vanishes. Here $\pi$ denotes the fundamental group at infinity of $X$ (or the fundamental groupoid, if $X$ has more than one end). Again $n \geq 6$, but if $\pi$ is not too complicated, $n$ can be 5 (see [5]).

In modern terminology, $\pi$ can be described as the fundamental group(oid) of the space, $\operatorname{Holink}(\hat{X}, \infty)$, where $\hat{X}$ denotes the one point compactification of $X$. This space, Holink $(X, A)$, (the "homotopy link" of $A$ in $X$ ), is defined 
for any subspace $A$ of a topological space $X$ as:

$$
\operatorname{Holink}(X, A)=\left\{\sigma \in \operatorname{Map}([0,1], X) \mid \sigma^{-1}(A)=0\right\} .
$$

It is given the compact-open topology. It comes with two maps,

$$
A \stackrel{p_{X}}{\longleftarrow} \operatorname{Holink}(X, A) \stackrel{j_{X}}{\longrightarrow} X-A: \quad j_{X}(\sigma)=\sigma(1), p_{X}(\sigma)=\sigma(0) .
$$

It is used, in [14], as a substitute for the normal bundle of $A$ in $X$.

F. Quinn recasts this subject of end theorems into a controlled context (see [11], [12], [14]). He considers a locally compact pair $(X, A)$, where $A$ is closed in $X, X-A$ is an $n$-manifold and $A$ is an ANR. He constructs a controlled, locally finite $K_{0}$-group, $\tilde{K}_{0}^{l f}\left(A, p_{X}\right)$, where $p_{X}: \operatorname{Holink}(X, A) \rightarrow$ $A$ is the projection. He then defines an element, $q_{0}(X, A) \in \tilde{K}_{0}^{l f}\left(A, p_{X}\right)$ whenever $A$ is tame in $X$. He shows that when $n \geq 6, \quad q_{0}(X, A)$ vanishes if and only if $A$ has a mapping cylinder neighborhood in $X$. We recall this key notion of tameness in 1.3 below. Also, as a service to the reader, we provide a short summary of the basic facts about the controlled end theorem in an Appendix.

1.2. Quinn's obstruction has the following appealing naturality property. Suppose $A$ is tame in $X$, and $X^{\prime}$ is an open set of $X$. Then $A^{\prime}:=X^{\prime} \cap A$ is tame in $X^{\prime}$ and $\iota^{*} q_{0}(X, A)=q_{0}\left(X^{\prime}, A^{\prime}\right)$ where $i^{*}: \tilde{K}_{0}^{l f}\left(A, p_{X}\right) \rightarrow \tilde{K}_{0}^{l f}\left(A^{\prime}, p_{X^{\prime}}\right)$ is the restriction map. (For more discussion on this see the Appendix). We can therefore localize both the obstruction and its containing group as follows.

For any point $a \in A$, define $K_{0}^{l f}\left(\left(A, p_{X}\right)_{(a)}\right)=\underline{\lim } K_{0}^{l f}\left(A^{\prime}, p_{X}^{\prime} \mid A^{\prime}\right)$ (the direct limit is over the $X$ - neighborhoods $X^{\prime}$, of $\left.a\right)$. Then the image of any one of the obstructions, $q_{0}\left(X^{\prime}, A^{\prime}\right)$ in $K_{0}^{l f}\left(\left(A, p_{X}\right)_{(a)}\right)$ is independent of the $X$-neighborhood $X^{\prime}$ chosen. We will write this image $q_{0}\left((X, A)_{(a)}\right)$.

1.3. Definition: A closed set $A$ in a topological space $X$ is tame in $X$ if:

1. A is forward tame. That is to say, there exists a neighborhood $U$ of $A$ in $X$ and a map $F: U \times[0,1] \rightarrow X$ such that $F^{-1}(A)=A \times[0,1] \cup U \times 0$ and $F(x, t)=x, \forall(x, t) \in A \times[0,1] \cup X \times 1$.

2. $A$ is reverse tame. That is to say, there is a map $R:(X-A) \times[0,1] \rightarrow$ $X-A$ so that: 
(a) $R(x, 0)=x, \forall x \in X-A$.

(b) for each $t \in(0,1], C l_{X} R((X-A) \times[t, 1]) \subset X-A$.

(c) the map, $R \cup 1_{A}:((X-A) \times[0,1]) \cup(A \times 0) \rightarrow(X-A) \cup(A)=X$ is continuous.

(In short, $X-A$ deforms promptly, but not abruptly, away from $A$, and moreover, a neighborhood of $A$ compresses reluctantly down to $A$ ).

1.4. The goal of this work is to prove an end theorem, analogous to that of Siebenmann's, but for a stratified space, the stratified analogue of a topological manifold. These were introduced by F. Quinn (in [14]) under the name "manifold homotopy stratified sets"; our terminology is due to Hughes [6] and Weinberger [20]).

A stratified space is a locally compact space, finitely filtered by closed subsets, $X=X^{n} \supset X^{n-1} \supset \ldots X^{-1}=\emptyset$. Each stratum $X_{i}:=X^{i}-X^{i-1}$ must be a manifold, and the boundary of $X$, defined by the rule, $\partial X:=$ $\cup_{i}\left(\partial X_{i}\right)$ must be closed in X. Moreover each $X_{i}$ must be tame in $X^{i} \cup X^{j}$ for each $j>i$. The projection, $\operatorname{Holink}\left(X_{i} \cup X_{j}, X_{i}\right) \stackrel{p}{\longrightarrow} X_{i}$ must be a fibration, and the inclusion $\left.\operatorname{Holink}\left(\partial X_{i} \cup \partial X_{j}, \partial X_{i}\right) \rightarrow \operatorname{Holink}\left(X_{i} \cup X_{j}, X_{i}\right)\right|_{\partial X_{i}}$ must be a fiber homotopy equivalence over $\partial X_{i}$.

Each open set of $X$ is again a stratified space, with the induced stratification. Each skeleton $X^{m}=X_{0} \cup X_{1} \cup \ldots \cup X_{m}$ is also a stratified space, as are $\partial X$ and $X / \partial X$. (The stratification on $X / \partial X$ is defined by requiring $(X / \partial X)_{i}=X_{i}-\partial\left(X_{i}\right)$ for each $\left.i>0\right)$. Quinn's Collaring Theorem (see [14]) says that $\partial X$ has a collar neighborhood in $X$, if $\partial\left(X^{5}\right)$ has a collar neighborhood in $X^{5}$. Finally, for any topological manifold $M, X \times M$ is a stratified space with the stratification induced from the one on $X$.

We begin with a stratified space $X$ with empty boundary. We seek to find a completion of $X$. By this we mean a compact stratified space $\bar{X}$ such that $X=\bar{X}-\partial \bar{X}$, and $\partial \bar{X}$ has a collar neighborhood in $\bar{X}$. The obvious necessary condition for this is that $X$ be tame ended. By this we mean simply that the one point compactification of $X, \hat{X}:=X \cup \infty$, is again a stratified space. Here the stratification is: $\hat{X}_{0}=X_{0} \cup \infty ; \quad \hat{X}_{j}=X_{j}, \forall j>0$. This is equivalent to saying that $\{\infty\}$ is tame in $X_{j} \cup\{\infty\}$ for each $j$. In particular, by reverse tameness each $X^{j}$ has only finitely many ends.

If a completion is impossible, we seek at least an exhaustion of $X$. By this we mean an increasing sequence of compact stratified subspaces of $X$, with bicollared boundaries in $X$, whose union is $X$. This also may be impossible. 
1.5. The End Obstruction. Let $X$ be a tame ended stratified space. For each $m>0$ we define,

$$
\gamma_{m}(X)=q_{0}\left(\left(\hat{X}^{m}, \hat{X}^{m-1}\right)_{(\infty)}\right) \in K_{0}^{l f}\left(\left(\hat{X}^{m-1}, p_{\hat{X}^{m}}\right)_{(\infty)}\right)
$$

where $p_{\hat{X}^{m}}: \operatorname{Holink}\left(\hat{X}^{m}, \hat{X}^{m-1}\right) \rightarrow \hat{X}^{m-1}$ is projection. We then set:

$$
\gamma_{*}(X)=\underset{m}{\oplus} \gamma_{m}(X) \in \underset{m}{\oplus} K_{0}^{l f}\left(\left(\hat{X}^{m-1}, p_{\hat{X}^{m}}\right)_{(\infty)}\right) .
$$

Our main results are:

1.6. Theorem. Suppose $X$ is a stratified space, with empty boundary, which admits a completion. Then $\gamma_{*}(X)=0$.

1.7. Theorem. Let $X$ be a tame ended stratified space with empty boundary. Let $A$ be any closed pure subset of $X$, containing $X^{5}$, such that $A$ admits a completion, $\bar{A}$. Suppose $\gamma_{*}(X)=0$. Then $X$ admits a completion $\bar{X}$ such that $C l_{\bar{X}}(A)=\bar{A}$.

(A pure subset is one which is the union of components of strata.)

1.8. Note Suppose the stratified space $X$ is an $n$-manifold. That is to say, $X^{n}=X$ and $X^{n-1}=\emptyset$. Then $U^{n-1}=\{\infty\}$, for each neighborhood $U$ of $\infty$ in $\hat{X}$. So $\gamma_{*}(X)$ reduces to $\gamma_{n}(X)$, and $\tilde{K}_{0}^{l f}\left(U^{n-1}, p_{U}\right)$ reduces to $\tilde{K}_{0}(\mathbb{Z} \pi)$, $\pi=\pi_{1}(\operatorname{Holink}(\hat{X}, \infty))$. In this case $\gamma_{*}(X)$ is Siebenmann's obstruction.

1.9. Note Following ([20], p.219), we say that a finite group action on a manifold, $(M, G)$ is a stratified $G$ manifold if the fixed set of each subgroup, $M^{H}$, is a manifold, and $M^{H}$ is locally flat in $M^{K}$ for each $K \subset H$. By ([14] $1.4,1.5$ and 1.6), this is equivalent to saying that $X=M / G$ is a stratified space when it is stratified by its orbit type components. Our main theorem implies immediately a similar theorem about $G$-manifolds:

Corollary 1.10. Let $(M, G)$ be a stratified $G$-manifold with $\partial M=\emptyset$. Then $(M, G)$ is the interior of a compact stratified $G$-manifold with collared boundary iff $X=M / G$ is tame ended, $\gamma_{*}(X)=0$, and $X^{5}$ has a completion.

1.11. Definition $\left(\right.$ of $\left.\left.K_{0}^{l f}\left(\left(\hat{X}^{n-1}, p_{\hat{X}}\right)_{(\infty)}\right)\right) \stackrel{\partial}{\rightarrow} \varliminf_{\longrightarrow} K_{0}^{l f}\left(W^{n-1}, p_{W}\right)\right)$

By an $\infty$-neighborhood in a locally compact space $X$, we mean a set of the form $W=U-\{\infty\}$, where $U$ is a neighborhood of $\infty$ in $\hat{X}$. 
Let $X$ be an $n$-dimensional stratified space. First suppose $X^{n-1}$ has a completion $Y$. Let $B=\partial Y$. Choose a collar neighborhood for $\partial Y$ in $Y$, say $B \times(0, \infty] \subset Y$. Then the open cone of $B, O B$ can be identified to $B \times(0, \infty] / B \times \infty$. The open cone becomes a neighborhood of $\infty$ in $\hat{X}^{n-1}$; moreover $\infty$ has a cofinal sequence of such neighborhoods, $B \times(k, \infty] / B \times \infty, k=0,1,2, \ldots$ The restriction maps connecting these are isomorphisms. This implies that the obstruction $\gamma_{*}(X)$ reduces to $\gamma_{n}(X)$, and moreover, that $\left.\tilde{K}_{0}^{l f}\left(\left(\hat{X}^{n-1}, p_{\hat{X}}\right)_{(\infty)}\right)\right)$ can be identified to $\tilde{K}_{0}^{l f}\left(O B,\left.p_{\hat{X}}\right|_{O B}\right)$, where $\left.\operatorname{Holink}\left(\hat{X}, \hat{X}^{n-1}\right)\right|_{O B} \stackrel{\left.p_{\hat{X}}\right|_{X B}}{\longrightarrow} O B$ is the projection map.

Now the fundamental group(oid) of the $n$ - stratum of the end of $X$ is defined as:

$$
\pi=\pi_{1}\left(\operatorname{Holink}\left(X_{n} \cup \infty, \infty\right)\right),
$$

where $X_{n} \cup \infty$ is topologized as a subspace of $\hat{X}$. We can analyze $\gamma_{n}(X) \in$ $\tilde{K}_{0}^{l f}(O B, p)$ further by considering the exact sequence induced by the cone point inclusion, $\{\infty\} \stackrel{\iota}{\longrightarrow} O B$ :

$$
\tilde{K}_{0}(\mathbb{Z} \pi) \stackrel{\imath_{*}}{\longrightarrow} \tilde{K}_{0}^{l f}(O B, p) \longrightarrow \tilde{K}_{0}^{l f}\left(B \times(0, \infty),\left.p\right|_{B \times(0, \infty)}\right) \longrightarrow \tilde{K}_{-1}(\mathbb{Z} \pi)
$$

Identify $B$ with $B \times 1 \subset B \times(0, \infty) \subset X^{n-1}$ and set $p_{B}=\left.p\right|_{B \times 1}$. The above sequence can be written as:

$$
\ldots \longrightarrow \tilde{K}_{0}(\mathbb{Z} \pi) \stackrel{\imath_{*}}{\longrightarrow} \tilde{K}_{0}^{l f}(l O B, p) \stackrel{\partial}{\longrightarrow} K_{-1}\left(B, p_{B}\right) \longrightarrow K_{-1}(\mathbb{Z} \pi) \ldots
$$

It turns out that $\partial \gamma_{n}(X) \in K_{-1}\left(B, p_{B}\right)$ is the obstruction to finding an exhaustion of $X$.

More generally now, for any tame ended stratified space $X$, we consider the inclusion $\{\infty\} \rightarrow U$, for each neighborhood $U$ of $\infty \in \hat{X}$. By taking the limit over all $\infty$-neighborhoods $W$, in $X$, we obtain an exact sequence generalizing the one above:

$$
\left.\rightarrow \tilde{K}_{0}(\mathbb{Z} \pi) \stackrel{\imath_{*}}{\longrightarrow} \tilde{K}_{0}^{l f}\left(\left(\hat{X}^{n-1}, p_{\hat{X}}\right)_{(\infty)}\right) \stackrel{\partial}{\longrightarrow} \varliminf_{\longrightarrow} K_{0}^{l f}\left(W^{n-1}, p_{W}\right)\right) \rightarrow \ldots
$$

We conjecture here that $\left.\lim _{B} K_{0}^{l f}\left(W^{n-1}, p_{W}\right)\right)=K_{-1}\left(B^{n-1}, p_{n}\right)$. Here $B^{m}:=B_{1} \cup \cdots \cup B_{m}$, where $\overrightarrow{B_{m}}:=\operatorname{Holink}\left(X_{m} \cup \infty,\{\infty\}\right) . B^{m}$ is topologized as a subset of $\operatorname{Holink}\left(\hat{X}^{m},\{\infty\}\right)$. The map $p_{m}$ is the pulback of $\operatorname{Holink}\left(X^{m}, X^{m-1}\right) \rightarrow X^{m-1}$ via the map $B^{m-1} \stackrel{j_{X^{m-1}}}{\longrightarrow} X^{m-1}$.

We now identify the obstruction to finding an exhaustion of $X$. 
Theorem 1.12 (Exhaustibility Theorem). Let $X$ be a tame ended stratified space with empty boundary. Assume that $X^{5}$ admits an exhaustion. Assume that $\partial \gamma_{n}(X)=0, \forall n \geq 6$. Then $X$ admits an exhaustion.

Conversely, suppose that $X$ admits an exhaustion, $\operatorname{dim}(X)=n$, and $X^{n-1}$ admits a completion. Assume all the fundamental groups of the fibers of the map, Holink $\left(X, X^{n-1}\right) \stackrel{p_{X}}{\rightarrow} X^{n-1}$ are good. Then $\partial \gamma_{n}(X)=0$.

1.13. We say that a group $G$ is good if $K_{i}(\mathbb{Z}[G])=0$ for $i \leq-2$. No example of a bad group is known. In fact any subgroup of a uniform discrete subgroup of a virtually connected Lie group is known to be good. (See [3]).

We conjecture that the $\partial \gamma_{*}(X)=0$ for all exhaustible stratified spaces.

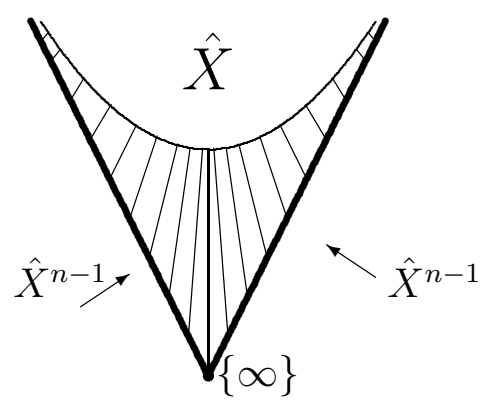

Figure 1: Neighborhood of $\infty$ in $\hat{X}$, when $\gamma_{*}(X)=0$

1.14. Note Suppose $K$ is a compact subset of a finitely filtered space $X$. Assume $X-K$ is a stratified space and $X$ is tame ended (in the sense that $\hat{X}-K$ is a stratified space). Then the conclusion and proof of Theorem 1.6, Theorem 1.7, and Theorem 1.12 are still valid for $X$, with only the most obvious changes.

Corollary 1.15. Let $X$ be a tame ended stratified space. Assume $X^{4} \times S^{1}$ has a completion. Then $X \times S^{1}$ has a completion.

Proof. Whenever Quinn's obstruction, $q_{0}(X, A)$, is defined, we know that $q_{0}\left(X \times S^{1}, A \times S^{1}\right)=0$. This can be seen as follows. Quinn's obstruction $q_{0}(X, A)$ is the limit of certain $\epsilon-$ controlled finiteness obstructions, 


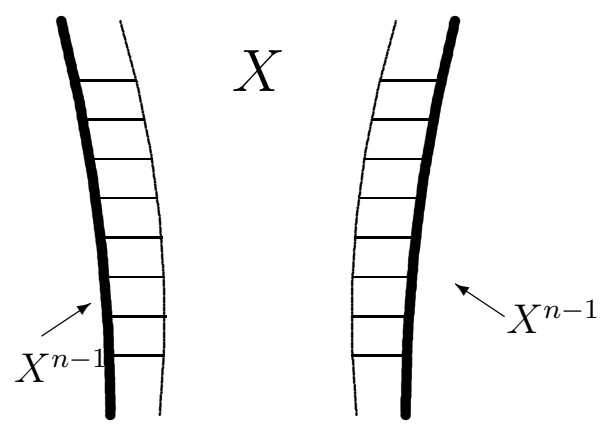

Figure 2: An $\infty$-neighborhood in $X$ when $\partial \gamma_{n}(X)=0$

$q_{0}(X, A ; \epsilon)$. The obstruction $q_{0}(X, A ; \epsilon)$ measures whether a neighborhood $N$ of the end of $X-A$, which one knows to be $\epsilon$-finitely dominated, can be made $4 \epsilon$ - homotopy equivalent to a finite complex. But the argument of Ferry [4] makes it obvious that $N \times S^{1}$ is $\epsilon$ homotopy equivalent to a finite complex, $K$, if measurements are still taken in $A$ (rather than in $A \times S^{1}$ ). The diameter of this homotopy equivalence when measured in $S^{1}$ might be large, say $D$. But upon passing to the $2^{n}$-fold cover of $S^{1}$, (which we can identify with $S^{1}$ ) and to the corresponding covers of $N \times S^{1}$ and $K$, we get an induced homotopy equivalence whose diameter in A is still $\epsilon$, while its diameter in $S^{1}$ has decreased to $D / 2^{n}$. This shows that $q_{0}\left(X \times S^{1}, A \times S^{1} ; \epsilon\right)=0$, for each $\epsilon$. In particular $q_{0}\left(X \times S^{1}, A \times S^{1}\right)=0$. Therefore, for any tame-ended stratified space, $\gamma_{*}\left(X \times S^{1}\right)=0$ and Theorem 1.7 implies the result.

One of our main tools for proving Theorem 1.7 is a transversality theorem which we believe will be of independent interest to topologists. This concerns an $n$-dimensional stratified space $X$, whose singular set, $X^{n-1}$, can be written in the form $X^{n-1}=N \times(-1,1)$, where $N$ is a stratified space. The goal is to write a neighborhood of $X^{n-1}$ in the same manner. We show that Quinn's mapping cylinder neighborhood obstruction, $q_{0}\left(X, X^{n-1}\right)$, is the only obstruction to achieving this goal. Here are the theorem and corollary:

Theorem 1.16 (Transversality Theorem). Let $X$ be a stratified space of dimension $n$. Assume that $\partial X=\emptyset$, and that $X^{n-1}=N \times(-1,1)$, for some stratified space $N$. Assume that $X^{n-1}$ has a mapping cylinder neighborhood 


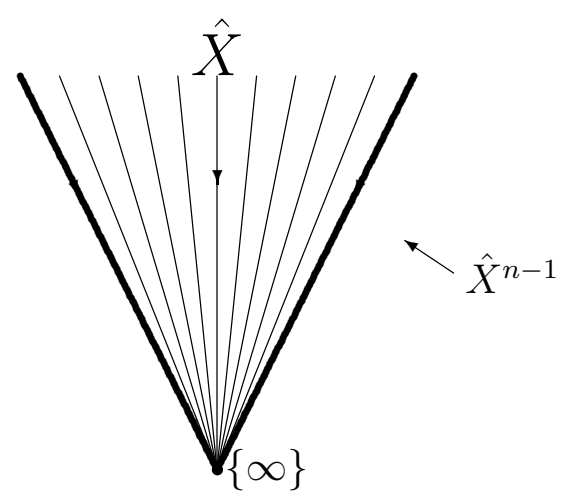

Figure 3: A neighborhood of $\infty$ when $X$ has a completion

in $X$. Let $C$ be a closed set of $X$ such that $C \cap X^{n-1} \subset N \times(-1,0)$. Then there are closed subsets $V, W$ of $X$ which are stratified subspaces of $X$, such that:

$$
\begin{gathered}
X=V \cup W ; \partial V=\partial W=V \cap W ; \\
N \times(-1,0]=V^{n-1} ; \quad N \times[0,1)=W^{n-1} ; C \subset V .
\end{gathered}
$$

(In the above theorem, we say $V$ is a stratified subspace of $X$ if the filtration on $V$ induced from that of $X$ makes $V$ into a stratified space. Note, then, that this does not imply that $\partial V \subset \partial X)$.

Corollary 1.17. Let $X$ be an $n$-dimensional stratified space with $n \geq 6$ and $\partial X=\emptyset$. Let $F: X \rightarrow \mathbb{R}$ be any continuous function for which $F \mid X^{n-1}$ : $X^{n-1} \rightarrow \mathbb{R}$ has 0 as a regular value. Let $\epsilon: X \rightarrow(0, \infty)$ be any continuous function. Let $N=F^{-1}(0) \cap X^{n-1}$. Then $q_{0}\left(\left(X, X^{n-1}\right)_{(N)}\right)=0$ if and only if there is a map $f: X \rightarrow \mathbb{R}$ for which $|f(x)-F(x)|<\epsilon(x)$, for all $x \in X$, $F\left|X^{n-1}=f\right| X^{n-1}$, and $f$ has 0 as a regular value.

(In the above Corollary, we say that 0 is a regular value of $f: X \rightarrow \mathbb{R}$, if there is an open neighborhood $U$ of $f^{-1}(0)$ and a commutative diagram :

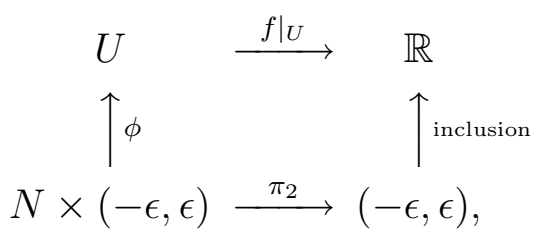


where $N$ is a stratified space, $\phi$ is an stratum preserving homeomorphism and $\pi_{2}$ is the second coordinate projection).

\subsection{Note}

Corollary 1.17 provides a topological equivariant transversality theorem for equivariant maps, $f: M \rightarrow \mathbb{R}$, when $(M, G)$ is a stratified $G$-manifold of dimension $n$, and $X=M / G$. It shows that if $\left(f^{H}\right) / N(H): M^{H} / N(H) \rightarrow \mathbb{R}$ already has 0 as a regular value for each subgroup $H \neq\{1\}$, then there is a single obstruction to approximating $f$ by a $G$ map transverse to 0 , which extends each $f^{H}$.

Note that the obstruction to doing so, $q_{0}\left(\left(M / G,(M / G)^{n-1}\right)_{(N)}\right)$ in 1.17, takes values in $K_{-1}\left(N, p_{X} \mid N\right)$, at least when $M$ is compact, because:

$$
\left.K_{-1}\left(N, p_{X} \mid N\right)=\tilde{K}_{0}^{l f}\left(N \times(-\epsilon, \epsilon), p_{X} \mid N \times(-\epsilon, \epsilon)\right)=\tilde{K}_{0}^{l f}\left(X^{n-1}, p_{X}\right)_{(N)}\right)
$$

if $N \times(-\epsilon, \epsilon)$ is a neighborhood of $X^{n-1} \cap\left(f^{-1}(0) / G\right)$ in $X^{n-1}$. Here

$$
N=\left\{x \in M: f(x)=0, \text { and } G_{x} \neq 1\right\} / G .
$$

This obstruction is simple to analyze when no fixed set has codimension 1 or 2 in any bigger fixed set. For in this case the fundamental group of the fiber of $\operatorname{Holink}\left(X, X^{n-1}\right) \rightarrow X^{n-1}$ over the point $G x / G \in X^{n-1}$ is $G_{x}$. Now $K_{i}(\mathbb{Z}[G])=0$ if $i \leq-2$, for any finite group $G$ (see [C]). So, by the spectral sequence of [12], we conclude that the obstruction group is:

$$
K_{-1}\left(N, p_{X} \mid N\right)=H_{0}\left(N ; K_{-1}\left(\mathbb{Z}\left[G_{x}\right]\right)\right)=\varliminf_{\longrightarrow} K_{-1}\left(\mathbb{Z}\left[G_{x}\right]\right) .
$$

Here the limit is taken over the poset given by the stratum components of $N$, ordered by reverse inclusion of their closures.

1.19. Here is an outline of the rest of this paper.

In section 2 we prove the Transversality theorem 1.16, and its corollary 1.17. They follow from a sharper but more technical Theorem 2.2. Perhaps the key result here is Lemma 2.5.

In section 3 we begin by proving Theorem 1.6. The proof is by no means immediate. The proof of Theorem 1.7 is then proved, relying heavily on Theorem 2.2. We then prove the Exhaustibility Theorem.

The first author wishes to thank both Jim West and Frank Quinn for raising the question of an "equivariant Siebenmann's theorem" some years ago, quite independently of each other, in informal conversations, in his presence. 


\section{The Transversality Theorem}

The goal of this section is to prove Theorem 1.16 and Corollary 1.17. We begin with a careful definition of a mapping cylinder neighborhood.

2.1. Definition (Mapping Cylinder Neighborhood) Let $A$ be a closed subset of a topological space $X$. A neighborhood $M$ of $A$ in $X$ is a mapping cylinder neighborhood if:

1. The frontier of $M$ in $X$, denoted $\partial M$, is a topological manifold.

2. There exists a continuous map $p: \partial M \rightarrow A$, and a homeomorphism from its mapping cylinder to $M$, say $f: C y l(p) \rightarrow M$, such that $\left.f\right|_{\partial M \cup A}=$ identity $\left.\right|_{\partial M \cup A}$.

The pair $(f, p)$ is called a cylinder structure for $M$.

Once $f$ and $p$ are chosen we write $[x, t]$ for the image of a point $(x, t) \in$ $\partial M \times[0,1]$ under the map $\partial M \times I \rightarrow C y l(p) \rightarrow M$. Usually, $p$ is surjective, so that each point of $M$ has this form, and $[x, t]=\left[x^{\prime}, t^{\prime}\right]$ iff $(x, t)=\left(x^{\prime}, t^{\prime}\right)$ or $t=t^{\prime}=0$ and $p(x)=p\left(x^{\prime}\right)$.

For a point $m=[x, t] \in M$, we call $x$ and $t$ its mapping cylinder coordinates.

Theorem 1.16 and Corollary 1.17 will follow quickly from the next result.

Theorem 2.2. Let $X$ be an $n$-dimensional stratified space with $\partial X=\emptyset$. Assume that $X^{n-1}=N \times(-1,1)$ for some stratified space $N$. Assume $M$ is a mapping cylinder neighborhood of $X^{n-1}$ in $X$, with cylinder structure $(p, f)$ and mapping cylinder coordinates $[x, t]$. Then there are stratified subspaces $V, W$ of $X$, such that:

i. $V$ and $W$ are closed subsets of $X$.

ii. $X=V \cup W ; \partial V=\partial W=V \cap W ;$

iii. $N \times(-1,0]=V^{n-1} ; N \times[0,1)=W^{n-1}$.

Moreover, if $p: \partial M \rightarrow N \times(-1,1)$ is surjective, with coordinates $\partial M \stackrel{n}{\rightarrow}$ $N, \partial N \stackrel{s}{\rightarrow}(-1,1)$, then $V$ and $W$ can be chosen as:

- $V=(X-\operatorname{Int} M) \cup\{[x, t] \in M \mid s(x) \leq t<1\}$, 
- $W=\{[x, t] \in M \mid 0 \leq t \leq s(x)\}$.

\subsection{Beginning of the proof of Theorem 2.2}

Let $X$ be as in the hypotheses of the theorem. Let $M$ be a mapping cylinder neighborhood for $X^{n-1}$ in $X$. Therefore $\partial M$ is a closed subset of $X$ and a topological manifold, $\partial M \cap X^{n-1}=\emptyset$, and there exists a map $p: \partial M \rightarrow X^{n-1}$, so that $M=C y l(p)$. Necessarily, by a result of B. Hughes, $p$ is a stratified approximate fibration (see [6], Prop. 2.1).

We can assume that $p$ is a surjective map - otherwise, we will replace $X$ by $C l_{X}\left(X-X^{n-1}\right)$. A point of $M$ can be written as $[x, t]$, where $x \in \partial M, t \in$ $[0,1]$. Notice that $[x, 0]=\left[x^{\prime}, 0\right]$ if and only if $p(x)=p\left(x^{\prime}\right)$. Write the map $p: \partial M \rightarrow N \times(-1,1)$ as $: p(x)=(n(x), s(x))$, where $n: \partial M \rightarrow N$ and $s: \partial M \rightarrow(-1,1)$.

The next simplification is to note that we need only prove Theorem 2.2 in the case when $X=M-\partial M$. To see this let $V_{M}$ and $W_{M}$ be the subsets of $M-\partial M$ obtained by applying Theorem 2.2 to $M-\partial M$. Then $W_{M}=$ $\{[x, t] \in M \mid 0 \leq t \leq s(x)\}$. Set $V=V_{M}$ and $W=X-\operatorname{Int}\left(V_{M}\right)$. Then $V$ and $W$ provide the required decomposition for $X$.

From now on we will replace $X$ by $M-\partial M$. Define:

- $V=\{[x, t] \in M \mid s(x) \leq t<1\}$

- $W=\{[x, t] \in M \mid 0 \leq t \leq s(x)\}$

- $U=V \cap W=\{[x, t] \in M \mid s(x)=t<1\}$

Note that $V \cup W=M-\partial M$. Theorem 2.2 is now reduced to proving:

2.4. Claim: $V$ and $W$ are stratified subspaces of $X$ ( it is clear that $\partial V=$ $U=\partial W)$.

To prove 2.4 we need:

2.5. Lemma. $U$ is a strong deformation retract of both $V$ and $W$. The deformations preserve strata.

Deferring the proof of Lemma 2.5 temporarily, we will show how Lemma 2.5 implies 2.4 and consequently Theorem 2.2. 


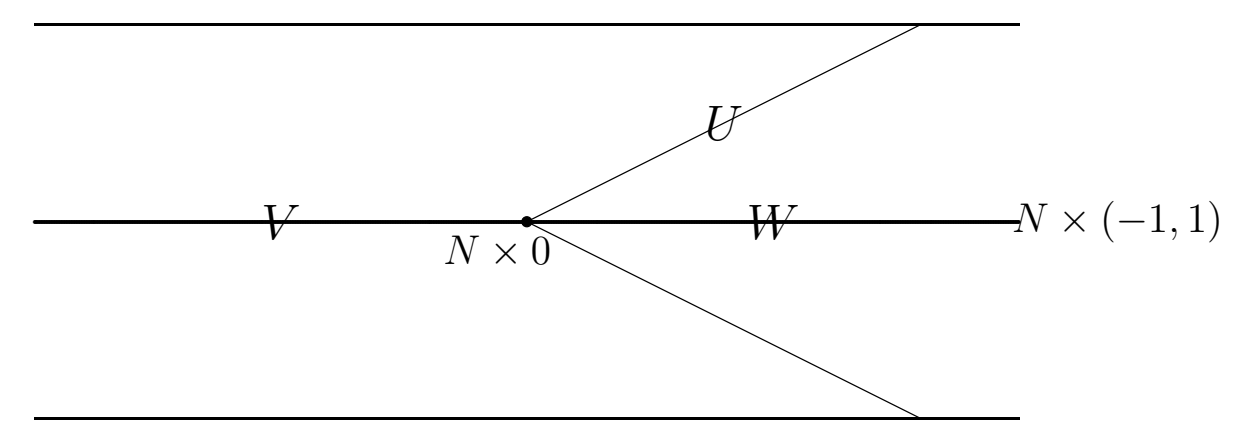

Figure 4: Mapping Cylinder Neighborhood of $N \times(-1,1)$

\subsection{Completion of the proof of Theorem 2.2}

The subset $U$ is obviously a closed set of $M-\partial M$. Since $U^{n-2}=N \times 0$, each stratum of $U^{n-2}$ is a manifold. Moreover, the top stratum, $U_{n-1}$, is homeomorphic to $p^{-1}(N \times(0,1))$, an open set of $\partial M$. This homeomorphism sends the point $[x, s(x)]$ to $x$. Next note that the $t$-coordinate of $M$ supplies a collaring for $U_{n-1}$ both in $V_{n}$ and in $W_{n}$. Therefore $V_{n}$ and $W_{n}$ are manifolds with common boundary $U_{n-1}$. Hence all strata of $V$ and $W$ are manifolds. Moreover $V^{n-1}$ and $W^{n-1}$ are stratified spaces with common boundary $U^{n-2}$. By Lemma 2.5 $U, V$ and $W$ are all stratified deformation retracts of $M-\partial M$. This implies that $U^{n-2}$ is forward and reverse tame in $U$, and that $\operatorname{Holink}\left(U, U^{n-2}\right)$ is a fiberwise deformation retract of $\operatorname{Holink}\left(M,\left.\left(M^{n-1}\right)\right|_{U^{n-2}}\right)$. The same holds for $V$ and $W$. This immediately implies that $V$ and $W$ are stratified spaces with common boundary $U$. This ends the proof of 2.4 and consequently Theorem 2.2 .

\subsection{Proof of Lemma 2.5}

Let $U^{\prime}, V^{\prime} \subset X^{n-1} \times[0,1]$ be defined as follows:

- $U^{\prime}=\{(n, s, t) \in N \times(-1,1) \times[0,1] \mid s=t\}$

- $V^{\prime}=\{(n, s, t) \in N \times(-1,1) \times[0,1] \mid s \leq t\}$

We first prove that $U$ is a stratified strong deformation retract of $V$.

There is obviously a strong, stratum preserving deformation retraction of $V^{\prime}$ down to $U^{\prime}$; we denote it by $g_{\lambda}: V^{\prime} \rightarrow V^{\prime}, 0 \leq \lambda \leq 1$, with $g_{0}=$ $1_{V^{\prime}}$ and $g_{1}\left(V^{\prime}\right)=U^{\prime}$. Namely: $g_{\lambda}(n, s, t)=(n, s+\lambda(t-s), t)$. The map 
$p \times 1: M-X^{n-1}=\partial M \times(0,1] \rightarrow X^{n-1} \times(0,1]$ is a stratified approximate fibration, because $p$ is. Now, $V-V^{n-1} \subset(p \times 1)^{-1}\left(V^{\prime}\right)$. So by choosing an approximate lifting of the deformation $\left\{g_{\lambda}\right\}_{0 \leq \lambda \leq 1}$, we obtain a stratum preserving deformation $G_{\lambda}: V \rightarrow V, 0 \leq \lambda \leq 1$ satisfying:

i. $G_{0}=\left.1_{V} \cdot G_{\lambda}\right|_{U}=1_{U}$, for any $\lambda$.

ii. Let $V_{+} \subset V$ be defined as:

$V_{+}=\{[x, t] \in M: 0<s(x) \leq t<1\} \cup\{[x, 0] \in M \mid s(x)=0\}$.

Then $G_{1}(V) \subset V_{+}$.

iii. Each $G_{\lambda}$ preserves the $t$-coordinate. That is to say, for each $[x, t]$ in $M$, there is a $y \in \partial M$ so that $G_{\lambda}([x, t])=[y, t]$.

iv. $\left.G_{\lambda}\right|_{V^{\prime}}=g_{\lambda}$ for each $\lambda$.

But $U$ is obviously a stratum preserving deformation retract of $V_{+}$(simply deform each point $[x, t]$ in $V_{+}$down to $\left.[x, s(x)]\right)$. The composite of these two deformations is a stratified deformation retraction of $V$ down to $U$.

We now prove that $U$ is a stratified strong deformation retract of $W$.

Let $h_{\lambda}: W^{n-1} \rightarrow M^{n-1}, 0 \leq \lambda<1$ be defined by: $h_{\lambda}(n, s)=(n, s-\lambda)$, for all $(n, s) \in N \times[0,1)$. An approximate lift of this deformation provides a deformation $H_{\lambda}: W \rightarrow W, 0 \leq \lambda \leq 1$ such that:

i. $H_{1}=\left.1_{W} \cdot H_{\lambda}\right|_{U}=1_{U}$, for all $\lambda$.

ii. Let $W^{-}=\left\{[x, t] \in M \mid 0 \leq \frac{s(x)}{2} \leq t \leq s(x)\right\} \subset W$. Then $H_{0}(W) \subset$ $W^{-}$.

Here are the messy analytic-geometry details of the construction of $H_{\lambda}$. Suppose $\bar{H}_{\lambda}: W-U \rightarrow M_{N}-M^{n-1}, 0 \leq \lambda \leq 1$ is a deformation of the identity inclusion $\bar{H}_{0}$, such that $\left.\bar{H}_{\lambda}\right|_{\sigma W}=h_{\lambda}$, and the following diagram $\epsilon$-commutes:

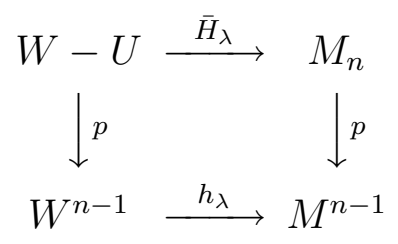


Here $\epsilon: W \rightarrow[0,1)$ is a continuous function, $\epsilon^{-1}(0)=W^{n-1}$ and the metric on $M^{n-1}=N \times(-1,1)$ is chosen so that $\pi_{2}: N \times(-1,1) \rightarrow(-1,1)$ is distance non-increasing.

Assume also that $\bar{H}_{\lambda}$ has the generic form $\bar{H}_{\lambda}([x, t])=[\phi(x, t), t]$. Then for any $[x, t] \in W-\left(W^{n-1} \cup U\right)$, we have $\bar{H}_{\lambda}([x, t]) \in W^{-}$, provided that $t+\epsilon \leq s(x)-\lambda \leq 2 t-\epsilon$. Now let $\lambda([x, t])=s(x)-t-\epsilon$. Note that $\bar{H}_{\lambda}([x, t]) \in W^{-}$, provided that $\epsilon \geq \frac{t}{2}$ and $\epsilon \leq s(x)-t$.

Set $\epsilon([x, t])=\frac{t}{2}(s(x)-t)$. Then $\frac{t}{2} \leq \epsilon \leq s(x)-t$. Notice that $\lambda([x, t])=$ 0 , if $[x, t] \in U$.

Hence we get the homotopy $H_{u}: W \rightarrow W, 0 \leq u \leq 1$ of the identity, by defining $\left.H_{u}\right|_{W n-U}=\left.\bar{H}_{u \lambda}\right|_{W n-U}$. We know that $H_{u} \mid U$ is the identity and also that $H_{u} \mid W^{n-1}=h_{u s}$.

Next note that $U$ is a stratified strong deformation retract of $W^{-}$(the deformation moves the point $[x, t]$ along the obvious path up to the point $[x, s(x)])$. This deformation, when composed with the deformation $\left\{H_{\lambda}\right\}$ above, gives a stratum-preserving deformation retraction of $W$ down to $U$. This completes the proof of Theorem 2.2.

The following corollary to Theorem 2.2 is an attempt to interpret the significance of the stratified space $W$ occuring in the proof of Theorem 2.2. We will use it in section 3 .

Corollary 2.8. Let $A$ be a stratified space. Let $M$ be the mapping cylinder of a map $p: \partial M \rightarrow A \times(-1,1)$, where $\partial M$ denotes an $n-1$ dimensional manifold. Filter $M$ by the rule:

$$
M_{n}=M-A \times(-1,1) ; \quad M^{n-1}=A \times(-1,1) \text { (as filtered spaces). }
$$

Assume $M$ is a stratified space. Let $\phi: p^{-1}(A \times[0,1)) \rightarrow[0,1)$ be any continuous map for which $\phi^{-1}(0)=p^{-1}(A \times 0)$. Let

$$
W_{\phi}=\{[x, t] \in M \mid p(x) \in A \times[0,1) \text {, and } t \leq \phi(x)\} .
$$

Then $W_{\phi}$ is a stratified subspace and a closed subset of $M . W_{\phi}-(A \times 0)$ is a mapping cylinder neighborhood of $A \times(0,1)$ in $M$.

Proof. $W_{\phi}$ is obviously a closed set of $M$. Also, $W_{\phi}$ is homeomorphic to the stratified space $W$ of Theorem 2.2. The homeomorphism sends any point $[x, t \phi(x)]$ of $W_{\phi}$ to the point $[x, t s(x)]$ of $W$. Since the homeomorphism preserves filtrations, the corollary then follows immediately from 2.2 . 


\subsection{Proof. of Theorem 1.16 and Corollary 1.17}

Let $X$ and $C$ be as in the Transversality Theorem 1.16. Let $M$ be a mapping cylinder neighborhood of $X^{n-1}$ in $X$ with cylinder structure $p$ : $\partial M \rightarrow X^{n-1}, f: C y l(p) \rightarrow M$. Set $W=\{[x, t] \in M \mid s(x) \leq t<1\}$ as in 2.2. By choosing the neighborhood $M$ sufficiently small, we can ensure that $W \cap C=\emptyset$. Then set $V=X-I n t W$. By Theorem 2.2, $V$ and $W$ are now as required to prove Theorem 1.16.

To prove 1.17, suppose first that $q_{0}\left(\left(X, X^{n-1}\right)_{(N)}\right)=0$. We can assume that some neighborhood of $F^{-1}(0)=N$ in $X^{n-1}$ has the form $N \times$ $(-\epsilon, \epsilon)$ and that this neighborhood has a mapping cylinder neighborhood in $X$. By Theorem 1.16, we can split $X$ as: $X=V \cup W$, where $V^{n-1}=$ $F^{(-1)}(-\infty, 0] ; W^{n-1}=F^{(-1)}([0, \infty))$ and where $V$ and $W$ are stratified subspaces with common boundary. By Quinn's collaring theorem,[14], the collars of $\partial\left(V^{n-1}\right)=\partial\left(W^{n-1}\right)$ in $V^{n-1}$ and $W^{n-1}$, given by the product structure on $N \times(-\delta, \delta)$, can be extended to collar neighborhoods of $V \cap W$ in $V$ and in $W$. So if $M=V \cap W=\partial V=\partial W$, we can assume that an open neighborhood $E$ of $M$, in $X$, has the form $M \times(-\delta, \delta)$, and that $F \mid E^{n-1}=\pi_{2}: N \times(-\delta, \delta) \rightarrow(-\delta, \delta)$. This implies that there is a continuous map $f^{\prime}: X \rightarrow \mathbb{R}$ such that $M=\left(f^{\prime}\right)^{-1}(0), f^{\prime}=F$ on $X^{n-1}$, and $f^{\prime}=\pi_{2}$ on $M \times\left(\delta^{\prime}, \delta^{\prime}\right)$ for some $\delta^{\prime}<\delta$. In particular, in some neighborhood $D$ of $X^{n-1},\left|f^{\prime}(x)-F(x)\right|<\epsilon(x)$ for all $x$ in $D$, and $f^{\prime}$ has 0 as a regular value. By the manifold-transversality theorem of Kirby and Siebenmann [7], there is a subneighborhood $C \subset D$ of $X^{n-1}$, and a continuous map $f^{\prime \prime}: X_{n} \rightarrow \mathbb{R}$ having 0 as a regular value such that $f^{\prime \prime}=f^{\prime}$ on $X_{n} \cap C$ and $\left|f^{\prime \prime}(x)-F(x)\right|<\epsilon(x)$ for all $x$ in $X_{n}$. Define $f: X \rightarrow \mathbb{R}$ by the rule: $f\left|X^{n-1}=F\right| X^{n-1}, f\left|X_{n}=f^{\prime \prime}\right| X_{n}$. Then $f$ has 0 as a regular value, and $|f(x)-F(x)|<\epsilon(x)$ for all $x$ in $X$. This proves one direction of Corollary 1.17 .

The other direction is even easier. Assume $X \stackrel{f}{\rightarrow} \mathbb{R}$ is a map having 0 as a regular value, and $f\left|X^{n-1}=F\right| X^{n-1}$. A neighborhood $X^{\prime}$ of $F^{-1}(0)$ then has the form $M \times(-1,1)$, where $M$ is a stratified subspace for which $N=M \cap X^{n-1}$. By 2.1, $q_{0}\left(X^{\prime}, X^{\prime}(n-1)\right)=0$. This implies at once, that $q_{0}\left(\left(X, X^{(n-1)}\right)_{(N)}\right)=0$. This completes the proof of 1.17 . 


\section{Proof of the Main Theorems}

Our first goal will be to prove Theorem 1.6. That is to say, we have to prove that the $\gamma$-invariant vanishes for any stratified space which admits a completion. We will need a sequence of lemmas to accomplish this.

Note: In sections 3.1-3.7 below, $X$ denotes a compact $n$-dimensional stratified space, and $A$ denotes $X^{n-1}$. We will write the cone on $X$ and the open cone on $X$ as follows:

$$
c X=X \times[0,1] / X \times 0 ; O X=X \times[0,1) / X \times 0 .
$$

So $X \times(0,1) \subset O X \subset c X$.

We define an induced stratified space structure on $c X$ by the rule: $(c X)^{n}=$ $c\left(X^{n-1}\right)$ for all $n \geq 0$. Then $O X$ is a stratified subspace of $c X$.

3.1. Lemma. For any $n \neq 3,4, A \times(0,1)$ has a mapping cylinder neighborhood in $X \times(0,1)$.

Proof. Since $A$ has a mapping cylinder neighborhood in $X$, if $n=1$ or 2 , by elementary arguments, we can assume $n \geq 5$. We have seen that $q_{0}\left(X \times S^{1}, A \times S^{1}\right)=0$, (see the proof of 1.15). By [12], $A \times S^{1}$ has a mapping cylinder neighborhod in $X \times S^{1}$, so the infinite cyclic cover of this neighborhood is the required neighborhood of $A \times(0,1)$ in $X \times(0,1)$.

3.2. Lemma. There is a mapping cylinder neighborhood $M$ of $A \times(0,1)$ in $X \times(0,1)$, and there are compact stratified spaces $U, V, W$, with the following properties.

a. $c X=V \cup W ; U=V \cap W ; U$ is a pure subset of $V$, and $U=\partial W$.

b. $c A \subset W \subset O X \cup c A ; \quad X \times 1 \subset V \subset c X-O A$.

c. For some compact set $K \subset O X, \quad M-K=(W \cap O X)-K$.

Note: The stratification on $V$ is not induced from $c X$.

Proof. Let $M_{2}$ be any mapping cylinder neighborhood of $A \times(0,2)$ in $X \times$ $(0,2)$. Here we use Lemma 3.1. We will assume $M_{2}=C y l(p)$, where $p$ : $\partial M(2) \rightarrow A \times(0,2)$. Choose a function $\phi: p^{-1}(A \times(0,1]) \rightarrow[0,1)$ with $\phi^{-1}(0)=p^{-1}(A \times 1)$, so that the space,

$$
W_{\phi}=\left\{[x, t] \in M_{2} \mid p(x) \in A \times(0,1], 0 \leq t \leq \phi(x)\right\}
$$




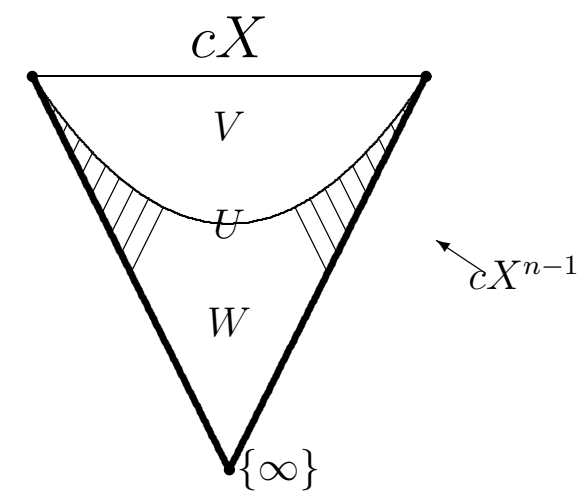

Figure 5: Picture of Lemma 3.2

is a subset of $X \times(0,1]$. Then by Corollary $2.8, W_{\phi}$ is a stratified subspace of $X \times(0,1]$, and $M:=W_{\phi}-A \times 1$ is a mapping cylinder neighborhood of $A \times(0,1)$ in $X \times(0,1)$. Now it is easy to see that $c A$ lies inside a compact stratified subspace $W$ of $(O X \cup c A)$ which is a neighborhood of $O A$ and for which $W-K=W_{\phi}-K$ for some compact $K \subset O X$. Set $U=\partial W$ (with the induced filtration), and $V=c X-\operatorname{Int}(W) . V$ is a stratified space, if we filter it by the rule:

$$
V_{n+1}=O X-W ; V^{n}=X \times 1 \cup \partial W ; V^{n-1}=A \times 1 \text { (as filtered spaces). }
$$

Now $M, U, V, W$ are as required.

3.3. Construction: Our goal here is to construct a specific strong deformation retraction of $\operatorname{Holink}(c X, c A)$ down to $\left.\operatorname{Holink}(c X, c A)\right|_{\{c\}}$.

For any map $I \stackrel{\phi}{\longrightarrow} I$ and any $t \in I$, define $I \stackrel{{ }^{t} \phi}{\longrightarrow} I$ by the rule:

$$
{ }^{t} \phi(s)=\phi(s)-(1-t) \min (\phi(0), \phi(s)) .
$$

For any map $I \stackrel{\psi}{\longrightarrow} X \times I$ and and $t \in I$, define $I \stackrel{{ }^{t} \psi}{\longrightarrow} X \times I$ by: ${ }^{t} \psi(s)=$ $\left(\psi_{1}(s),{ }^{t} \psi_{2}(s)\right)$.

Now define a strong deformation retraction, $\left\{F_{t}: 0 \leq t \leq 1\right\}$, of $\operatorname{Holink}(c X, c A)$ down to $\left.\operatorname{Holink}(c X, c A)\right|_{\{c\}}$ by the rule:

$$
F_{t}(\sigma)= \begin{cases}{ }^{t} \sigma & \text { if } \sigma \in \operatorname{Holink}(c X, c A)-\left.\operatorname{Holink}(c X, c A)\right|_{\{c\}} \\ \sigma & \text { if }\left.\sigma \in \operatorname{Holink}(c X, c A)\right|_{\{c\}}\end{cases}
$$


This deformation has the following obvious properties:

a. $F_{1}=$ identity ;

b. $\left.F_{0}(\operatorname{Holink}(c X, c A)) \subset \operatorname{Holink}(c X, c A)\right|_{\{c\}}$;

c. $F_{t}$ is a fiber preserving map covering the map $c A \stackrel{f_{t}}{\longrightarrow} c A$ given by: $f_{t}(a, s)=(a, s t)$, for $a \in A$ and $s \in[0,1]$;

d. $\left.F_{t}\left(\left.\operatorname{Holink}(c X, c A)\right|_{A \times 1}\right) \subset \operatorname{Holink}(O X, O A)\right|_{A \times t}$, if $0 \leq t \leq 1$.

e. The above formula also provides a homotopy $\left\{F_{t}^{\prime}: 0 \leq t \leq 1\right\}$, of the inclusion, $\operatorname{Map}(I, c X-\{c\}) \rightarrow \operatorname{Map}(I, c X)$ to a map

$$
F_{0}^{\prime}: \operatorname{Map}(I, c X-\{c\}) \rightarrow \operatorname{Holink}(c X, c) .
$$

Let $\pi$ denote the fundamental groupoid of $O X-O A$. We can identify $\pi$ with the fundamental groupoid of the fiber of $\operatorname{Holink}(O X, O A) \stackrel{p_{O X}}{\rightarrow} O A$ over $c$. Then the inclusion $\{c\} \stackrel{i}{\rightarrow} O A$ induces a map,

$$
\tilde{K}_{0}(\mathbb{Z}[\pi])=\tilde{K}_{0}\left(\{c\}, p_{O X} \mid\{c\}\right) \stackrel{i_{*}}{\rightarrow} \tilde{K}_{0}^{l f}\left(O A, p_{O X}\right) .
$$

The spaces $X_{n} \times 1$ and $V_{n+1}$ are finitely dominated, and so these have finiteness obstructions in $\tilde{K}_{0}(\mathbb{Z} \pi)$ denoted $\sigma\left(X_{n} \times 1\right)$ and $\sigma\left(V_{n+1}\right)$.

Next look at the chain complex, $\left.C_{*}\left(W_{n+1} \cup(\partial W)_{n},(\partial W)_{n}\right)\right)$. This is a complex of geometric modules on $E:=H \operatorname{olink}(O X, O A)$ and its class is the element $q_{0}(O X, O A) \in \tilde{K}_{0}^{l f}\left(O A, p_{O X}\right)$.

3.4. Lemma. $q_{0}(O X, O A)=i_{*}\left(\sigma\left(X_{n} \times 1\right)-\sigma\left(V_{n+1}\right)\right)$.

Proof. Near $A \times 1, W-(A \times 1)$ has the structure of a mapping cylinder neighborhood over $A \times(0,1)$ (see Lemma 3.2c). Therefore, the class $\left.\left[C_{*}\left(W_{n+1} \cup(\partial W)_{n},(\partial W)_{n}\right)\right)\right]$ can be viewed as the image of an element of $\tilde{K}_{0}\left(O A, p_{O X}\right)$ under the natural map $\tilde{K}_{0}\left(O A, p_{O X}\right) \rightarrow \tilde{K}_{0}^{l f}\left(O A, p_{O X}\right)$. But the inclusion induced map, $\tilde{K}_{0}\left(\{c\}, p_{O X} \mid\{c\}\right) \rightarrow \tilde{K}_{0}\left(O A, p_{O X}\right)$ is an isomorphism by 3.3. This means that:

$$
q_{0}(O X, O A)=\left[C_{*}\left(W_{n+1} \cup(\partial W)_{n},(\partial W)_{n}\right)\right]=i_{*} \sigma\left(W_{n+1} \cup(\partial W)_{n},(\partial W)_{n}\right) .
$$


Here the pair $\left(W_{n+1} \cup(\partial W)_{n},(\partial W)_{n}\right)$ is relatively finitely dominated because of its mapping cylinder neighborhood structure near $A \times 1$, and the fact that $O A$ is tame in $O X$. So by excision,

$$
\begin{gathered}
q_{0}(O X, O A)=i_{*}\left(\sigma\left(W_{n+1} \cup(\partial W)_{n},(\partial W)_{n}\right)=i_{*}(\sigma(O X-O A, V \cap O X))\right. \\
=i_{*}(\sigma(O X-O A)-\sigma(V \cap O X))=i_{*}\left(\sigma\left(X_{n} \times 1\right)-\sigma\left(V_{n+1}\right)\right) .
\end{gathered}
$$

3.5. Lemma. The classes $\sigma\left(V_{n+1}\right)$ and $\sigma\left(X_{n}\right)$ are in the image of the homomorphism, $\tilde{K}_{0}\left(A \times 1, p_{c X \mid A \times 1}\right) \stackrel{\left(F_{0}\right)_{*}}{\longrightarrow} \tilde{K}_{0}(\mathbb{Z}[\pi])$, induced by the map:

$$
\left.\left.\operatorname{Holink}(c X, c A)\right|_{A \times 1} \stackrel{F_{0}}{\rightarrow} \operatorname{Holink}(O X, O A)\right|_{\{c\}} .
$$

Proof. According to [14], the composite map $\operatorname{Holink}(X, A) \stackrel{j_{X}}{\rightarrow} X-A \rightarrow$ $O X-O A$ induces a homomorphism, $\left(j_{X}^{\prime}\right)_{*}: \tilde{K}_{0}\left(A, p_{X}\right) \rightarrow \tilde{K}_{0}(\mathbb{Z}[\pi])$ satisfying, $\left(j_{X}^{\prime}\right)_{*} q_{0}(X, A)=\sigma\left(X_{n}\right)$. This proves $\sigma\left(X_{n} \times 1\right) \in \operatorname{Im}\left(\left(F_{0}\right)_{*}\right)$.

Similarly $\sigma\left(V_{n+1}\right)=F_{0 *}^{\prime}\left(q_{0}\left(V, V^{n}\right)\right)$, where $F_{0 *}^{\prime}: \tilde{K}_{0}\left(V^{n}, p_{V}\right) \rightarrow \tilde{K}_{0}(\mathbb{Z}[\pi])$ is induced by the map, $\operatorname{Holink}\left(V, V^{n}\right) \stackrel{F_{0}^{\prime}}{\longrightarrow} \operatorname{Holink}(O X, O A) \mid\{c\}$.

Now the fiber of $\operatorname{Holink}\left(V, V^{n}\right) \rightarrow V^{n}$ is contractible over any point of $V_{n}$. This is because $V_{n+1} \cup V_{n}$ is a manifold with boundary; its boundary is $V_{n}$. Therefore the inclusion, $A \times 1 \rightarrow V^{n}$ induces an isomorphism, $\tilde{K}_{0}(A \times$ $\left.1, p_{V} \mid(A \times 1)\right) \stackrel{i_{*}^{\prime}}{\rightarrow} \tilde{K}_{0}\left(V^{n}, p_{V}\right)$. In particular, then, $q_{0}\left(V, V^{n}\right) \in \operatorname{Im}\left(i_{*}^{\prime}\right)$, and so $\sigma\left(V_{n+1}\right) \in \operatorname{Im}\left(\left(F_{0}^{\prime}\right)_{*} i_{*}^{\prime}\right) \subset \operatorname{Im}\left(\left(F_{0}\right)_{*}\right)$ as required.

3.6. Lemma. Let $\tilde{K}_{0}\left(A \times 1,\left.p_{c A}\right|_{A \times 1}\right) \stackrel{\ell_{*}}{\rightarrow} \tilde{K}_{0}^{l f}\left(O A, p_{O X}\right)$ be induced by the composite map $\ell$ given by:

$$
\left.\left.\operatorname{Holink}(c X, c A)\right|_{A \times 1} \stackrel{F_{0}}{\rightarrow} \operatorname{Holink}(O X, O A)\right|_{\{c\}} \stackrel{\imath}{\rightarrow} \operatorname{Holink}(O X, O A),
$$

where $\imath$ denotes the inclusion. Then $\ell_{*}=0$.

Proof. Because $F_{0}$ is fiber homotopic to $F_{t}$ for any $0<t<1$, the map $\ell_{*}$ is also induced by the map:

$$
\left.\left.\operatorname{Holink}(c X, c A)\right|_{A \times 1}\right)\left.\stackrel{F_{t}}{\rightarrow} \operatorname{Holink}(O X, O A)\right|_{A \times[t, 1)} \stackrel{\text { incl. }}{\rightarrow} \operatorname{Holink}(O X, O A) .
$$

This shows that $\ell_{*}$ factors through $\tilde{K}_{0}^{l f}\left(A \times[t, 1),\left.p_{O X}\right|_{A \times[t, 1)}\right)$. This last group is zero by the usual Eilenberg swindle argument. So $\ell_{*}=0$. 
3.7. Lemma. $q_{0}(O X, O A)=0$.

Proof. By Lemma 3.5, $i_{*}\left(\sigma\left(V_{n+1}\right)\right)$ and $i_{*}\left(\sigma\left(X_{n} \times 1\right)\right)$ lie in $\operatorname{Im}\left(\ell_{*}\right)$, which is zero by Lemma 3.6. But therefore, by Lemma 3.4, $q_{0}(O X, O A)=0$

\subsection{Proof of Theorem $\mathbf{1 . 6}$}

Let $X$ be a stratified space without boundary, which admits a completion , say $\bar{X}$. We have to prove that $q_{0}\left(\left(\hat{X}^{n}, \hat{X}^{n-1}\right)_{(\infty)}\right)=0$ for each $n$. Let $B=\partial \bar{X}$. $\infty$ has an open neighborhood in $\hat{X}^{n}$ of the form $O B^{n-1}$. But by Lemma 3.7, $q_{0}\left(O B^{n-1}, O B^{n-2}\right)=0$. The image of $q_{0}\left(O B^{n-1}, O B^{n-2}\right)$ in $\tilde{K}_{0}^{l f}\left(\left(\hat{X}^{n-1}, p_{X^{n}}\right)_{(\infty)}\right)$ is $\gamma_{n}(X)$. (See the definition of $\gamma_{n}(X)$ in 1.4 and 1.1). Therefore, $\gamma_{n}(X)=0$ for all $n$. This completes the proof of Theorem 1.6.

Proof of Theorem 1.7.

Suppose $X$ is an $n$-dimensional tame ended stratified space, and $\gamma_{*}(X)=$ 0 . Assume also that a pure closed subset $A$,containing $X^{5}$ has a completion, $\bar{A}$. Working inductively we will assume that $X^{n-1} \supset A$, and that $X^{n-1}$ has an $\infty$-neighborhood of the form $B \times[0, \infty)$, so that $X^{n-1}$ has a completion, with collared boundary $B$, where $B$ is some compact stratified space .

(An $\infty$-neighborhood in a locally compact space $X$ is a set of the form $N-\{\infty\}$ where $N$ is a neighborhood of $\infty$ in $\hat{X}$.)

We must prove that there is a compact stratified space $C$ for which $B=C^{n-2}$, together with a homeomorphism of stratified spaces onto an $\infty$ - neighborhood of $X$ of the form $C \times[1, \infty) \stackrel{h}{\longrightarrow} X$ so that $\left.h\right|_{B \times[1, \infty)}=$ identity $\left.\right|_{B \times[1, \infty)}: B \times[1, \infty) \rightarrow X^{n-1}$.

We begin by choosing any sequence $\mathcal{O}(0), \mathcal{O}(1), \ldots \mathcal{O}(k), \ldots$ of open neighborhoods of $\infty$ in $\hat{X}$ such that $\mathcal{O}(k)^{n-1}=B \times(k, \infty)$ for each $k$. Because $\gamma_{n}(X)=0$ we can choose a mapping cylinder neighborhood $M(0)$ of $\mathcal{O}(0)^{n-1}$ in $\mathcal{O}(0)$, at least if $\mathcal{O}(0)$ is chosen small enough.

We now apply Corollary 2.8 to each of the sets $\mathcal{O}(k)$ to produce, inductively, a sequence $\{M(k)\}_{k=1}^{\infty}$, where $M(k)$ is a mapping cylinder neighborhood of $\mathcal{O}(k)^{n-1}$ in $\mathcal{O}(k)$ so that:

a. $W(k):=(M(k)-\infty) \cup(B \times k)$ is a closed set of $X$ and a stratified subspace of $X$;

b. $\operatorname{Int}(W(k)) \supset W(k+1)$, for all $\mathrm{k}$. 


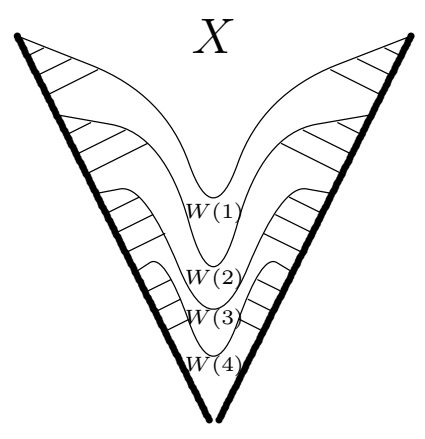

Figure 6: Neighborhoods of $\infty$ in $\hat{X}$

Now $\partial\left(W(k)^{n-1}\right)=B \times k$ is a strong stratum-preserving deformation retract of $W(k)^{n-1}=B \times[k, \infty)$, for each $\mathrm{k}$. Also $\partial\left(W(k)_{n}\right)$ is a deformation retract of $W(k)_{n}$. In fact the mapping cylinder lines give the deformation retraction. Therefore the inclusion $\partial W(k) \rightarrow W(k)$ induces a homotopy equivalence on each stratum. This implies that $\partial W(k)$ is a stratified strong deformation retract of $W(k)$.

It follows that for each $k \geq 1, V(k):=W(k)-\operatorname{Int}(W(k+1))$ is an h-cobordism of stratified spaces from $\partial W(k)$ to $\partial W(k+1)$, and a product cobordism on $V(k)^{n-1}=B \times[k, k+1]$.

We now apply Quinn's thin h-cobordism theorem $([12], \S 1.2)$. The obstruction to extending the product structure on $V(k)^{n-1}$ to a product structure on $V(k)$ lies in the controlled K-theory group $\tilde{K}_{1}^{l f}\left(O B, p_{k}\right)$, where

$$
\operatorname{Holink}(M(k) \cup \infty, B \times(k, \infty) \cup\{\infty\}) \stackrel{p_{k}}{\rightarrow} B \times(k, \infty) \cup\{\infty\}=O B
$$

is the projection (see [12], $§ 1.2$ or [14]). Moreover any element of $\tilde{K}_{1}^{l f}(O B, p)$ can be realized as the torsion of an h-cobordism $U=W(k)-W^{\prime}(k)$, where $W^{\prime}(k)$ is a stratified subspace of $W(k)$, such that $W^{\prime}(k)^{n-1}=W(k)^{n-1}=$ $B \times[k, \infty)$ and $W(k+1) \subset \subset W^{\prime}(k)-B \times k \subset \operatorname{Int}(W(k))$. Therefore we can, if necessary, replace $W(k)$ by $W^{\prime}(k)$, for suitable $W^{\prime}(k)$, so that $V(k)$ above can be given a product structure extending the one on $V(k)^{n-1}=B \times[k-1, k]$.

Now, $W(1)=\bigcup_{j>1} V(j)$ admits a homeomorphism

$$
\left(W(1), W(1)^{n-1}\right) \stackrel{h}{\longrightarrow} \partial W(1) \times[1, \infty)
$$


whose restriction to $B \times[1, \infty)$ is the identity. So, take $C=\partial W(1)$ and $h$ are as required and Theorem 1.7 is proved.

3.9. Proof of the Exhaustion Theorem. First we assume that $X^{5}$ admits an exhaustion and

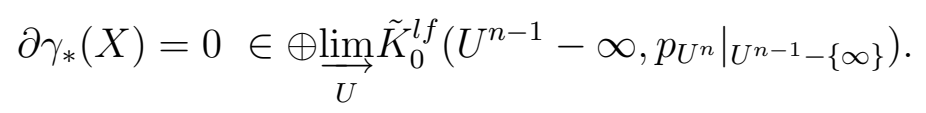

The limit is over neighborhoods $U$ of $\infty$ in $\hat{X}$. We must show that $X$ admits an exhaustion. Let $n=\operatorname{dim}(X)$. We can assume that $n \geq 6$, and that $X^{n-1}$ admits an exhaustion.

Let $K$ be a compact set of $X$ We must show there is a compact stratified subspace $V$ of $X$ such that $K \subset V$ and $\partial V$ is bicollared in $X$. Because $\partial \gamma_{n}(X)=0$, there is an $\infty$ - neighborhood $E$, in $X$, such that $q_{0}\left(E, E^{n-1}\right)=$ 0 . By induction there is a compact stratified subspace with bicollared boundary, say $V^{n-1}$, of $X^{n-1}$ such that $K^{n-1} \cup(X-E)^{n-1} \subset \operatorname{Int}\left(V^{n-1}\right)$. Set $N=\partial V^{n-1}$, and choose a neighborhood of $N$ in $E^{n-1}$ of the form $N \times(-1,1)$. Choose any proper map $F: X \rightarrow \mathbb{R}$, such that $V^{n-1}=X^{n-1} \cap F^{-1}((-\infty, 0])$, $K \subset F^{-1}((-\infty, 0])$, and $F \mid N \times(-\delta, \delta)=\pi_{2}: N \times(-\delta, \delta) \rightarrow(-\delta, \delta)$. Since $q_{0}\left(E, E^{n-1}\right)=0, N \times(-1,1)$ has a mapping cylinder neighborhood in $E$. Now apply Theorem 1.16 to obtain a proper map $f: X \rightarrow \mathbb{R}$ having 0 as a regular value such that $f\left|X^{n-1}=F\right| X^{n-1}$, and $K \subset f^{-1}((-\infty, 0))$. Then $V:=f^{-1}((-\infty, 0])$ is a compact stratified subspace of $X$, with bicollared boundary, such that $K \subset V$ as required.

Conversely, we now assume that $X^{n-1}$ has an $\infty$ - neighborhood of the form $B \times(0, \infty)$, and that $X$ has an exhaustion. We have to prove that $\partial \gamma_{*}(X)=0$. By the hypotheses, we can find a compact stratified subspace $N$ of $X$ with bicollared boundary, $\partial N$, such that $N^{n-1} \cup B \times(0, \infty)=X^{n-1}$. Let $M=\partial\left(N^{n-1}\right) \subset B \times(0, \infty)$. $\partial N$ has a neighborhood of the form $\partial N \times(-\varepsilon, \varepsilon)$ in $B \times(0, \infty)$.

Choose open sets $X^{\prime}, X^{\prime \prime}$ in $X$, so that $X^{\prime} \cap X^{n-1}=B \times(0, \infty), \quad X^{\prime \prime}=$ $\partial N \times(-\varepsilon, \varepsilon)$. The inclusion induces a restriction map,

$K_{-1}\left(B ; p_{X}\right)=\tilde{K}_{0}^{l f}\left(B \times(0, \infty) ; p_{X}\right) \stackrel{i^{*}}{\longrightarrow} \tilde{K}_{0}^{l f}\left(M \times(-\varepsilon, \varepsilon) ; p_{X}\right)=K_{-1}\left(M ; p_{X}\right)$.

Now $K_{-1}\left(B ; p_{X}\right)=\underset{[b] \in[B]}{\lim _{\longrightarrow}} K_{-1}\left(\mathbb{Z} G_{[b]}\right) ; K_{-1}\left(M ; p_{X}\right)=\underset{[m] \in[M]}{\lim _{[M}} K_{-1}\left(\mathbb{Z} G_{[m]}\right)$, by the good-holinks condition and the Quinn spectral sequence. Here the 
stratum components of $B$ form a poset, $[B]$ in which there is one morphism from $[b]$ to $\left[b^{\prime}\right]$ if $\left[b^{\prime}\right]$ is in the closure of the stratum component $[b]$; otherwise there is no morphism. The analogous rule holds for $[M]$. The map,

$$
\underset{[b] \in[B]}{\lim _{[m]}} K_{-1}\left(\mathbb{Z} G_{[b]}\right) \stackrel{i^{*}}{\longrightarrow} \underset{[m] \in[M]}{\lim } K_{-1}\left(\mathbb{Z} G_{[m]}\right)
$$

is the restriction of the map $\prod_{[b] \in[B]} K_{-1}\left(\mathbb{Z} G_{[b]}\right) \rightarrow \prod_{[m] \in[M]} K_{-1}\left(\mathbb{Z} G_{[m]}\right)$ which sends $x \in K_{-1}\left(\mathbb{Z} G_{[b]}\right)$ to the tuple whose coordinate in $K_{-1}\left(\mathbb{Z} G_{[m]}\right)$ is the image of $x$, if $[m]$ is in the closure of the stratum component of $B \times(0, \infty)$ containing [b]; in all other coordinates this tuple is zero. But this map is obviously injective since each stratum component of $B \times(0, \infty)$ meets $M$. Hence $i^{*}$ is injective. But $i^{*} q_{0}\left(X^{\prime}, B \times(0, \infty)\right)=q_{0}\left(X^{\prime \prime}, M \times(-\varepsilon, \varepsilon)\right)=0$, since $X^{\prime \prime}=\partial N \times(-\varepsilon, \varepsilon)$. Therefore $q_{0}\left(X^{\prime}, B \times(0, \infty)\right)=0$, which implies that $\partial \gamma_{*}(X)=0$. This completes the proof of the exhaustion theorem.

3.10. Proof of Theorem 1.10. Let $X=M / G$, where $(M, G)$ is a stratified $G$-manifold, and $X$ is tame ended, with $\gamma_{*}(X)=0$. Then $X$ admits a completion. The boundary, $B$, of this completion admits a collar neighborhood. This gives an $\infty$-neighborhood in $X$ of the form $B \times(0, \infty)$. This neighborhood therefore has the form $N \times(0, \infty) / G$, where $N \times(0, \infty)$ is an $\infty$-neighborhood of $M$ for which $G$ acts trivially on the second coordinate, and $N / G=B$. Note that $N$ and each fixed set of $N$ is a homology manifold. Each fixed set of $N$ is 1-lc embedded in each bigger fixed set, unless the codemension is 2 ; in this case it is locally homotopically unknotted. This is clear because $N \times(0, \infty)$ is a stratified G-manifold. Each stratum of $N$ is a manifold. It follows immediately then, from [12] (Theorem 3.4.1, p.291) that $\mathrm{N}$ is a stratified G-manifold. This shows that $X \cup_{N \times(0, \infty)} N \times(0, \infty]$ is a compact stratified $G$-manifold, with collared boundary, whose interior is $X$.

3.11. There are stratified $G$-manifolds which are not exhaustable, but are tame ended. In fact, there is a semifree action of $G=\mathbb{Z} / 6 \mathbb{Z}$ on $M_{1}^{\prime}=$ $\mathbb{R}^{2 n+1}-\{0\}, n \geq 2$ with fixed set $\mathbb{R}^{1}-\{0\}$, for which $\partial \gamma_{2 n+1}(M / G) \neq 0$ in $K_{-1}(\mathbb{Z} G) \oplus K_{-1}(\mathbb{Z} G) \oplus K_{-1}(\mathbb{Z} G) \oplus K_{-1}(\mathbb{Z} G)$. Furthermore if $M_{1}=S^{2 n} \times S^{1}$ and $M_{1}^{\prime} \stackrel{\pi}{\longrightarrow} M_{1}$ is the usual covering map, then $\pi$ is equivariant with respect to a stratified $G$-action on $M_{1}$. This $G$-manifold, $\left(M_{1}, G\right)$ is h-cobordant (stratified and equivariant) to some $\left(M_{0}, G\right)$, whose universal cover $\left(M_{0}^{\prime}, G\right)$ has the form $(V-\{0\}, G)$, where $(V, G)$ is a linear representation of $G$. 
This example and the question of realizability of the obstruction $\gamma_{*}(X)$ are exposed in [18]. 


\section{A APPENDIX: A Summary of Controlled End Theorems}

Because the material on controlled end theorems is widely considered to make difficult reading, it seems to us to be appropriate to provide a brief yet explicit summary of the main ideas in this appendix.

A.1. The most important obstruction groups used by Quinn [11],[12], [14] and others (e.g. [10]) are $\tilde{K}_{1}^{l f}(B, p)$ and $\tilde{K}_{0}^{l f}(B, p)$, where $B$ is a locally compact metric space and $E \stackrel{p}{\longrightarrow} B$ is a continuous map. One writes these as $\tilde{K}_{0}(B, p)$ when $B$ is compact. These are defined by

$$
\tilde{K}_{i}^{l f}(B, p)=\varliminf_{\varepsilon} K_{i}^{l f}(B, p, \varepsilon)
$$

where the limit is taken over all continuous functions $\varepsilon: B \rightarrow(0, \infty)$. The groups $\tilde{K}_{i}^{l f}(B, p, \varepsilon)$ are defined in terms of the additive category of geometric modules and maps on $E$, which are locally finite over $B$. The constructions are worked out very carefully in [16]. When $B$ is compact, the geometric modules involved must perforce be compact, and we then write the group as $\tilde{K}_{i}(B, p, \varepsilon)$. More generally, for any $B$, if we restrict ourselves to finite geometric modules, the resulting group is $\tilde{K}_{i}(B, p, \varepsilon)$

A.2. Briefly, $\tilde{K}_{1}^{l f}(B, p, \varepsilon)$ is the set of $4 \varepsilon$-stable equivalence classes of $\varepsilon$ contractible chain complexes of such modules. To obtain such a contractible complex imagine some h-cobordism $\left(W ; V_{0}, V_{1}\right)$ between paracompact manifolds, equipped with a map $W \stackrel{f}{\longrightarrow} E$, so that $p f: W \rightarrow B$ is proper and the deformation of $W$, down to $V_{0}$ or $V_{1}$ has diameter less than $\varepsilon$ when measured in $B$. The chain complex $C$, of a handle decomposition for $\left(W, V_{0}\right)$, if chosen sufficiently fine, provides such a contractible complex whose class is independent of the handle structure and decomposition. See [11] and [12], especially [11], Ch.7. So the element $[C] \in \tilde{K}_{1}^{l f}(B, p, \varepsilon)$, obtained for each $\varepsilon$ defines a class $\tau\left(W ; V_{0}, V_{1}\right) \in \tilde{K}_{1}^{l f}(B, p)$ called the torsion of $\left(W ; V_{0}, V_{1}\right)$, provided that the diameters of the deformations can be chosen arbitrarily small. As in [9], every element of $\tilde{K}_{1}^{l f}(B, p)$ can be realized as the torsion of an h-cobordism on $V_{0}$; moreover if $\tau\left(W ; V_{0}, V_{1}\right)=0$, then there is a product structure (of small diamater) on $\left(W ; V_{0}, V_{1}\right)$. See [12] for details.

A.3. $\tilde{K}_{0}^{l f}(B, p ; \varepsilon)$ is defined (in [16], for example) as the set of $4 \varepsilon$-stable equivalence classes of chain complexes of $\varepsilon$-projective geometric modules. An $\varepsilon$-projective geometric module consists of a geometric module on $(B, p)$ 
together with an endomorphism, $p: M \rightarrow M$ such that $p^{2} \sim_{\varepsilon} p$ (see [16] for more details).

To get an element of $\tilde{K}_{0}^{l f}(B, p ; \varepsilon)$ imagine a chain complex $C$ of ordinary geometric modules, together with a chain map $C \stackrel{f}{\longrightarrow} C$ and a chain homotopy $h$ from $f^{2}$ to $F$, so that both $f$ and $h$ have small diameter. Then [15] and [16] give a explicit functorial construction which, from the input $(C, f, h)$ yields as output an $\varepsilon$-projective chain complex as required. We write $[C, f, h] \in \tilde{K}_{0}^{l f}(B, p ; \varepsilon)$ for the class of this complex.

A.4. To obtain such a triple $(C, f, h)$, suppose that $B$ above is a closed tame subset of a locally compact space $X$, and that $X-B$ is a manifold. Let $E=H \operatorname{link}(X, B)$. Let $M \cup B$ be a small closed neighborhood of $B$ in $X$, chosen so that $M$ is a manifold whose boundary is the frontier of $M \cup B$ in $X$, and so that the map $\partial M \rightarrow M \rightarrow E \stackrel{p}{\rightarrow} B$ is proper. The map $M \rightarrow E$ is defined by the forward tameness structure on $B$ in $X$.

Choose a handle decomposition on $(M, \partial M)$ so that the diameter of the handles approaches zero as one approaches $B$. The chain complex $C(M, \partial M)$ then has the following salient feature: for each $\varepsilon: B \rightarrow(0, \infty)$ there is a chain homotopy $H_{\varepsilon}$ of the identity to a map $F_{\varepsilon}: C(M, \partial M) \rightarrow C(M, \partial M)$ so that $F_{\varepsilon}(C(M, \partial M))$ is supported over a subset $K_{\varepsilon} \subset M$ for which $K_{\varepsilon} \rightarrow$ $M \rightarrow E \rightarrow B$ is a proper map. Moreover the diameter of $H_{\varepsilon}$ is less than $\varepsilon$, when measured in $B$. If $L \subset M$ is any preassigned subset for which $C l_{X}(L) \subset X-B$, then $H_{\varepsilon}$ can be chosen so that it is zero over $L$ and its image is supported over $X-L$. All of this is a consequence of the reverse tameness of $B$ in $X$.

Now, for each $\varepsilon$, let $C_{\varepsilon}$ denote the subcomplex of $C(M, \partial M)$ spanned by those handles meeting $K_{\varepsilon}$. Then $H_{\varepsilon}$ and $F_{\varepsilon}$ restrict to a triple $\left(C_{\varepsilon}, f_{\varepsilon}, h_{e p s}\right)$ as above, and the classes $\left[C_{\varepsilon}, f_{\varepsilon}, h_{\varepsilon}\right]$ combine to specify an element in $\tilde{K}_{0}^{l f}(B, p)$ which does not depend on the choice of the handle decomposition on $M$. This is the definition of $q_{0}(X, B)$.

(N.B. Quinn's definition of $q_{0}(X, B)$ is hard to locate. It occurs on page 408 of [12]).

A.5. The main geometric result of [11],[12] is that, with suitable hypotheses, $q_{0}(X, B)=0$ iff $B$ has a mapping cylinder neighborhood in $X$. See [12], Thm. 1.1 and [14],Thm. 1.7.

A.6. Here is a systematic approach to the controlled end obstruction of Quinn, motivated by A.3 and A.4. Let $E \stackrel{p}{\longrightarrow} B$ be as before. Let $C$ 
be a chain complex of geometric modules on $E \times[0, \infty)$, locally finite over $B \times[0, \infty)$.

We say $C$ is controllable over $p$, if for each $\varepsilon$, one can find a pair $\left(C_{\varepsilon}, H_{\varepsilon}\right)$ where $C_{\varepsilon}$ is a subcomplex of $C$, locally finite over $B$, and $H_{\varepsilon}$ is an $\varepsilon$ chain homotopy (when measurements are made in $B$ ), from $1_{C}$ to a chain map $F_{\varepsilon}: C \rightarrow C$ of the form $C \stackrel{f_{\varepsilon}}{\longrightarrow} C_{\varepsilon} \stackrel{i_{\varepsilon}}{\longrightarrow} C$, where $i_{\varepsilon}$ denotes inclusion.

We assume that each closed $\varepsilon$-ball in $B$ is compact. This ensures that $H_{\varepsilon} \mid C_{0}: C_{0} \rightarrow C$, is proper over $B$, for each subcomplex $C_{0}$ of $C$ which is locally finite over $B$. Therefore the triple $\left(C_{\varepsilon}, f_{\varepsilon} \mid C_{\varepsilon},\left(f_{\varepsilon} \circ H_{\varepsilon} \mid C_{\varepsilon}\right)\right)$ defines, as in A.3, an element $q(C ; \varepsilon) \in \tilde{K}_{0}^{l f}(B, p ; \varepsilon)$ which does not depend on the choice of $C_{\varepsilon}$. If $C_{\varepsilon}$ is fixed, this element $q(C ; \varepsilon)$ is independent of the choice of the chain homotopy $H_{\varepsilon}$ as well. So these elements $q(C ; \varepsilon)$ combine to define an element $q(C) \in \tilde{K}_{0}^{l f}(B, p)$ depending only on $C$. One sees easily that $q(C)=q\left(C / C_{0}\right)$ for any proper subcomplex $C_{0}$ of $C$.

Moreover, if $(M, \partial M)$ is as in A.4 and we choose any Urysohn function $\phi: X \rightarrow[0, \infty]$ so that $\phi^{-1}(0)=\partial M, \phi^{-1}(\infty)=B$, then $C(M, \partial M)$ becomes a controllable complex over $p$, and $q(C(M, \partial M))=q_{0}(X, B)$.

It is also not hard to prove that every element of $\tilde{K}_{0}^{l f}(B, p)$ has the form $q(C)$ for some controllable complex $C$.

A.7. The main homotopy theoretic result of [12] is that the groups $\tilde{K}_{i}(B, p)$ can be interpreted as homology groups of $B$, with stratified coefficients. For this, the required input is that $E \stackrel{p}{\longrightarrow} B$ be a stratified system of fibrations (see [12], Ch. 8). We make almost no use of this result however. (The exception is the proof of the second part of the exhaustion theorem).

A.8. Here we want to give a simple description of the restriction map used in 1.1, based on the discussion in A.6 above.

Let $X$ be a locally compact space. Let $B$ be a tame closed subspace, such that $X-B$ is a manifold. Suppose $X^{\prime}$ is an open set of $X$, and $B^{\prime}:=X^{\prime} \cap B$. Set $E^{\prime}=\operatorname{Holink}\left(X^{\prime}, B^{\prime}\right)$, and $p^{\prime}=\left.p\right|_{E^{\prime}}$. Then the definition of the restriction map $\iota^{*}: \tilde{K}_{0}^{l f}(B, p) \rightarrow \tilde{K}_{0}^{l f}\left(B^{\prime}, p^{\prime}\right)$ is obvious if, following Quinn, one interprets the groups $\tilde{K}_{i}^{l f}$ ( ) as a generalised locally finite homology theory as in A.7. But a more simple approach is available. Namely, suppose that $C$ is a controllable chain complex over $E \stackrel{p}{\longrightarrow} B$. Let $C_{0}$ be the subcomplex spanned by those basis elements which do not lie over $E^{\prime}$. Then $C^{\prime}:=C / C_{0}$ is controllable over $p^{\prime}$ because $C$ is controllable over $p$. The element $\iota^{*} q(C)$ is simply $q\left(C^{\prime}\right)$. Let $M$ be a manifold with boundary in $X-B$ such that 
$M \cup B$ is a neighborhood of $B$ in $X$. Set $C=C_{*}(M, \partial M)$. If $M^{\prime} \cup B^{\prime}$ is a manifold neighborhood of $B^{\prime}$ in $X^{\prime} \cap M$, and $C^{\prime}:=C\left(M^{\prime} . \partial M^{\prime}\right)$ we conclude that and $i^{*} q_{0}(X, B)=i^{*} q(C)=q\left(C^{\prime}\right)=q_{0}\left(X^{\prime}, B^{\prime}\right)$. 


\section{References}

[1] Browder, W. ; Levine, J. ; Livesay, G. R.: Finding a boundary for an open manifold Amer. J. Math. 871965 1017-1028.

[2] Carter, David W. :Lower K-theory of finite groups Comm. Algebra 8 (1980), no. 20, 1927-1937.

[3] Farrell, F. T. ; Jones, L. E.: The lower algebraic K-theory of virtually infinite cyclic groups, J. Jour. of K-Theory 9 (1995), no. 1, 13-30

[4] Ferry, Steve :A simple-homotopy approach to the finiteness obstruction Shape theory and geometric topology (Dubrovnik, 1981), pp. 73-81, Lecture Notes in Math., 870, Springer, Berlin-New York, 1981.

[5] Freedman, Michael H. ; Quinn, Frank:Topology of 4-manifolds Princeton Mathematical Series, 39. Princeton University Press, Princeton, NJ, 1990.

[6] Hughes Bruce C. :Geometric Topology of Stratified Spaces, preprint.

[7] Kirby, Robion C.; Siebenmann, Laurence C.: Foundational essays on topological manifolds, smoothings, and triangulations Annals of Mathematics Studies, No. 88. Princeton University Press, Princeton, N.J.; University of Tokyo Press, Tokyo, 1977.

[8] Miller, R. T. :Mapping cylinder neighborhoods of some ANR's Ann. of Math. (2) 103 (1976), no. 2, 417-427.

[9] Milnor, John: Lectures on the h-cobordism theorem Notes by L. Siebenmann and J. Sondow Princeton University Press, Princeton, N.J. 1965.

[10] Pedersen, Erik Kjaer :On the bounded and thin h-cobordism theorem parameterized by $\mathbf{R}^{k}$ Transformation groups, Poznań 1985, 306-320, Lecture Notes in Math., 1217, Springer, Berlin-New York, 1986.

[11] Quinn, Frank: Ends of maps. I. Ann. of Math. (2) 110 (1979), no. 2, 275-331.

[12] Quinn, Frank: Ends of maps. II. Invent. Math. 68 (1982), no. 3, 353-424. 
[13] Quinn, Frank: Ends of maps. IV. Controlled pseudoisotopy Amer. J. Math. 108 (1986), no. 5, 1139-1161.

[14] Quinn,Frank: Homotopically stratified sets J. Amer. Math. Soc. 1 (1988), no. 2, 441-499.

[15] Ranicki, Andrew:The algebraic theory of finiteness obstruction. Math. Scand. 57 (1985), no. 1, 105-126.

[16] Ranicki, Andrew ; Yamasaki, Masayuki: Controlled K-theory Topology Appl. 61 (1995), no. 1, 1-59.

[17] Siebenmann, Laurence C.: Obstructions to finding a boundary for an open manifold:: Thesis, Princeton University 1965.

[18] Vajiac, Bogdan: A controlled end theorem for stratified spaces, Ph.D. Thesis, in preparation.

[19] Wall, C.T.C.: Surgery on Compact Manifolds. New York, Academic Press 1970.

[20] Weinberger, Shmuel :The Topological Classification of Stratified Spaces Chicago Lectures in Mathematics, Chicago University Press 1994. 